\title{
Computer simulation study of pattern transfer in $A B$ diblock copolymer film adsorbed on a heterogeneous surface
}

\author{
A. Jayaraman, C. K. Hall, ${ }^{\text {a) }}$ and J. Genzer \\ Department of Chemical and Biomolecular Engineering, North Carolina State University, \\ College of Engineering 1, Box 7905, 911 Partners Way, Raleigh, North Carolina 27695
}

(Received 1 April 2005; accepted 3 August 2005; published online 23 September 2005)

\begin{abstract}
In this work we investigate how a pattern imposed in a copolymer film at a certain distance from the surface propagates through the film onto an adsorbing heterogeneous surface. We bias the copolymer film to adopt a specified target pattern and then use simulation to design a surface pattern that helps the adsorbed film to maintain that target pattern. We examine the effect of varying the copolymer chain length, the size of the target pattern, and the distance from the surface where the target pattern is applied, $z^{\prime}$, on the extent of pattern transfer. For each chain length, target pattern, and $z^{\prime}$ we compare the energy of the system when a pattern is applied in the bulk to the energy when no pattern is applied in order to understand why a certain pattern size is transferred to the surface with higher fidelity than the others. At constant chain length, pattern transfer is best when the pattern size brings the energy of the system close to the energy when no pattern is applied. At constant pattern size, pattern transfer is best in the systems with longer chains. This is because longer chains are more likely to adsorb as brushes and loops which then helps transfer the pattern through the adsorbed film down to the surface. (C) 2005 American Institute of Physics.

[DOI: 10.1063/1.2043048]
\end{abstract}

\section{INTRODUCTION}

Adsorption of polymers on solid surfaces plays an important role in many technological and scientific applications including adhesion, ${ }^{1} \quad$ chromatography, ${ }^{2}$ nanoscale patterning, ${ }^{3}$ biomedical implant modification, ${ }^{4,5}$ etc. In order to exploit these applications, it is important to understand how polymers organize in the vicinity of surfaces. Most surfaces in nature are physically or/and chemically heterogeneous and many polymers are typically composed of two or more types of monomers arranged in a specified sequence (diblock, triblock, or alternating copolymers) or a random sequence. Therefore, studying the adsorption of heteropolymers on heterogeneous surfaces is useful in describing real world situations.

There have been many experimental efforts aimed at understanding how chemically heterogeneous patterns on surfaces induce ordering in adsorbed block copolymers. ${ }^{6-12}$ Rockford et al. ${ }^{8,9}$ studied adsorption of polystyrenepoly(methyl methacrylate) (PS-PMMA) block copolymers on surfaces containing alternating stripes of gold and silicon oxide. The PS-PMMA block copolymers formed ordered lamellar microdomains oriented perpendicular to the surface with the PS domains covering the gold stripes and the PMMA domains covering the silicon oxide stripes. Rockford et al. ${ }^{8,9}$ and Kim et al. ${ }^{10}$ also found that the influence of the surface pattern on the lamellar ordering and orientation of adsorbed polymers is strongly dependent on the degree of commensurability between the polymer length and the stripe widths. Yang et al. ${ }^{11}$ found that when the width of the surface pattern is much greater than the bulk lamellar period of the

${ }^{a)}$ Electronic mail: hall@eos.ncsu.edu block copolymer then the lamellae orient parallel to the surface and replicate the underlying substrate pattern into the bulk. However, when the width of the surface pattern is commensurate with the bulk lamellar period of the block copolymer, the lamellae orient perpendicular to the substrate. Heier et al. ${ }^{12}$ investigated the behavior of symmetric poly(styrene2-vinylpyridine) (PS-PVP) block copolymer thin films on patterned substrates formed using microcontact printing that had alternating stripes of $\mathrm{H}_{3} \mathrm{C}$-and $\mathrm{HO}$-terminated selfassembled monolayers (SAMs). They found that the energy required to form islands of PS-PVP is different for each surface stripe. Since the $\mathrm{H}_{3} \mathrm{C}$-terminated SAM domains are energetically more favorable for the formation of islands than the HO-terminated SAM domains are, PS-PVP forms islands over the $\mathrm{H}_{3} \mathrm{C}$-terminated SAM domains.

In addition to the experimental work mentioned above, theory and simulation have been used to shed light on the organization of block copolymer bulk on patterned surfaces. ${ }^{13-24}$ Petera and Muthukumar used mean-field theory ${ }^{13}$ and self-consistent-field theory ${ }^{14}$ to show that the orientation of the lamellae formed by diblock copolymer depends on the ratio of the substrate pattern period $(L)$ and the radius of gyration of the diblock copolymer $\left(R_{g}\right)$ in a disordered melt. When $R_{g} / L \sim \sqrt{ }(2 \sqrt{ } 3) / 2 \pi$, the surface pattern period and the lamellar period are commensurate and the lamellae align perpendicular to the surface. Genzer used a three-dimensional (3D) self-consistent-field model to investigate the adsorption of copolymers from a copolymerhomopolymer mixture ${ }^{15-17}$ onto a heterogeneous surface composed of two chemically different regions arranged either in checkerboard or random patterns. He demonstrated that if heteropolymers recognize the patterns on the hetero- 
geneous surface, they can transcribe the pattern from the surface into three dimensions. Semler and Genzer used the bond fluctuation model and Monte Carlo simulations to study the effect of domain size ${ }^{18}$ and interaction potential ${ }^{19}$ on adsorption of diblock and alternating copolymers on chemically heterogeneous surfaces. The block copolymers recognize the surface pattern and transfer it to the bulk if the size of the adsorbing surface domain is commensurate with the parallel component of the radius of gyration of the block copolymers. The alternating copolymers recognize chemical patterns of sizes much smaller than the $R_{g, \|}$, the radius of gyration parallel to the surface of the copolymer. As the strength of attraction between the block copolymer and surface increases, the extent to which the pattern is transferred to the bulk remains unaffected. As the strength of attraction between the alternating copolymer and surface increases, the pattern transferred into the bulk at increasing distances from the surface resembles an inverted surface pattern. Jayaraman et al. developed a simulation method recently to design surfaces for recognizing specific monomer sequences in copolymers. $^{20}$ For copolymers with less blocky sequences, the designed surfaces recognize the correct sequence well when the segment-surface interactions dominate over the intersegment interactions. For copolymers with more blocky sequences, recognition is good when the segment-surface interactions are only slightly stronger than the intersegment interactions. Using a theoretical model, Kriksin et al. also found that when copolymers made of short blocks adsorb on heterogeneous surfaces, marked pattern recognition is possible at high polymer/surface attraction. ${ }^{21}$

All of the work done so far has dealt with understanding how the pattern on the heterogeneous surface gets recognized and propagates through the copolymer film. Here we attempt to understand how a pattern applied in the copolymer film at a certain distance from the surface propagates through the adsorbed film onto the adsorbing heterogeneous surface. Ultimately we intend to determine what surface pattern is required to induce a desired pattern in the adsorbed polymer film.

We use lattice Monte Carlo simulations to explore how the length of the diblock copolymer, size of the patterns (checkerboard), and the distance from the surface where the pattern originally exists affect the extent of pattern transfer through the adsorbed layers to the surface. A study of how a diblock copolymer film transfers patterns with sizes on the order of the copolymer length scale through the adsorbed layers could help us understand how to form unique nanoscale structures which in turn would be useful in nanolithography and the development of photonic devices. ${ }^{6,7}$

Our system consists of symmetric $A B$ diblock copolymers adsorbing onto a flat, chemically heterogeneous surface containing two types of sites: 1 and 2 . Our approach comprises several steps. First we bias the copolymer bulk to adopt a target pattern at a specified distance from the surface. Then we let the surface reorganize itself in response to the pattern in the copolymer bulk. We achieve this by switching the identities of sites on the surface and performing chain moves during the simulation with an acceptance criterion that minimizes the energy and biases the adsorbed layers to maintain the target pattern at the desired distance from the surface. We consider an attractive potential between like copolymer segments $(A-A$ and $B-B)$ and an attractive potential between the surface sites and copolymer segments $(A-1$ and $B-2)$. These potentials favor the transfer of the target pattern through the different adsorbed layers to the surface and help the surface to adopt a pattern commensurate to the target pattern. The more the surface resembles the target pattern, the higher the extent of pattern transfer through the adsorbed layers. We study three different chain lengths $(12,18$, and 24) and three different pattern sizes (checkerboard pattern of sizes $6 \times 6,12 \times 12$, and $24 \times 24)$. The pattern is applied at three different distances from the surface $\left(z^{\prime}=1,2\right.$, and 3$)$. We also compare each of these systems with the case when no pattern is applied while all other parameters are maintained the same. When no pattern is applied in the adsorbed layers, the copolymer chains are free to attain their natural conformations. We do this comparison to understand why a certain pattern size gets transferred to the surface better than the other patterns.

The highlights of our results are the following. As we increase the distance from the surface where the target pattern is applied, the pattern transfer to the surface decreases for all patterns and all chain lengths. For a constant pattern size, the pattern transfer to the surface increases as the chain length increases. For a constant chain length, the patterns that help the chains attain energies close to the case when no pattern is applied get transferred to the surface well. To test if we can use the designed surfaces from this stage and induce the target pattern back in the adsorbed layers of a new copolymer bulk, in the next stage we let a new copolymer bulk in a random configuration adsorb onto each of these "designed" surfaces. For all the pattern sizes and chain lengths considered, we do not see the transfer of the surface pattern back to the adsorbed layers and the copolymer chains form other energetically favorable patterns.

Section II introduces the simulation technique. Section III describes the application of this method to a system of block copolymers to learn how variations in copolymer chain length, target pattern size, and distance from the surface at which the pattern is applied affect the extent of pattern transfer from the bulk to the surface. A brief summary of our conclusions is given in Sec. IV.

\section{MODEL AND METHODS}

\section{A. Simulation technique}

We use lattice Monte Carlo (MC) simulation, which has the advantage that it is computationally fast and yet provides a good description of the large scale conformational features of polymer chains. Each MC simulation is performed on a cubic lattice in the $N V T$ ensemble, i.e., the total number of monomers in the simulation box, the volume of the simulation box, and the temperature of the system are fixed. The simulation box has periodic boundary conditions in the $x$ and $y$ directions. The dimensions of the simulation box in the $x$, $y$, and $z$ directions are 48,48 , and 12 , respectively. In the $z$ direction, there is an athermal surface at $z=13$ and an adsorbing surface at $z=0$. 
A system of symmetric diblock copolymers is in contact with a heterogeneous surface located at $z=0$. The diblock copolymers contain two types of segments: $A$ and $B$. The $A B$ diblock copolymer chains are modeled as self-avoiding walks on a cubic lattice. The surface contains two types of sites, 1 and 2. The values of the segment-segment interaction potentials, $\epsilon_{A A}, \epsilon_{B B}$, and $\epsilon_{A B}$, and the segment-surface interaction potential, $\epsilon_{A 1}, \epsilon_{B 1}, \epsilon_{A 2}$, and $\epsilon_{B 2}$, are assigned such that $\epsilon_{A A}, \epsilon_{B B}, \epsilon_{A 1}$, and $\epsilon_{B 2}$ are all negative (implying attractive potentials) and $\epsilon_{A B}, \epsilon_{A 2}$, and $\epsilon_{B 1}$ are zero. Attractive segmentsurface interactions, $\epsilon_{A 1}$ and $\epsilon_{B 2}$, cause the chains to adsorb on the surface. Attractive segment-segment interactions, $\epsilon_{A A}$ and $\epsilon_{B B}$, cause the adsorbed chains to pull other chains towards themselves leading to dense adsorbed layers near the surface. The segment-segment interaction potential is short ranged; it only affects nonbonded nearest neighbors. The segment-surface interaction potential is also short ranged, acting only between the sites on the adsorbing surface $(z=0$ plane $)$ and the segments that are on the plane adjacent to the adsorbing surface ( $z=1$ plane).

The volume fraction $\Phi$ of the $A B$ diblock copolymer segments in the simulation box is 0.35 . The interaction potentials are taken to be $\epsilon_{A 1}=\epsilon_{B 2}=-1.33 k T$ and $\epsilon_{A A}=\epsilon_{B B}=-0.6 k T$. These interaction potentials were chosen to be strong enough to cause the chains to adsorb onto the surface yet weak enough to avoid ordering or segregation of adsorbed chains. Having strong interaction between the copolymer segments and the surface helps the copolymer chains to overcome the entropic penalty associated with coil formation during adsorption.

We design the surface by interchanging the positions of the surface sites over the course of the simulation. This is accomplished by generating moves that switch the identity of the different sites on the surface in response to a target function that specifies the target pattern at a particular layer in the bulk. The target patterns in this paper are checkerboards of alternating regions of segments of type $A$ and type $B$. In addition to the surface site switches, we move the segments on the chain using the standard chain moves: kink jump (25\%), crankshaft (25\%), and reptation (50\%). The type of move and chain and segment numbers along the chain are all picked randomly. In one Monte Carlo step (MC step) we make $N_{\text {chains }} \times N_{\text {beads }}$ trials, where $N_{\text {chains }}$ is the number of chains in the simulation box and $N_{\text {beads }}$ is the number of segments in each chain. The acceptance criterion for the chain moves and the surface site switches are based on a modified Metropolis algorithm derived from reverse Monte Carlo acceptance criterion. ${ }^{25-27}$ The chain moves or site switches are accepted with a probability $P_{\text {acc }}$ given by

$$
\begin{aligned}
P_{\text {acc }}= & \min \left[1, \exp \left\{-\frac{1}{k T}\left[\left(U_{\text {new }}-U_{\text {old }}\right)\right.\right.\right. \\
& \left.\left.\left.+\frac{1}{T_{\Gamma} / T}\left(\Gamma_{\text {new }}-\Gamma_{\text {old }}\right)\right]\right\}\right],
\end{aligned}
$$

where the subscript old signifies the present state of the system, the subscript new signifies the new state of system after the chain move or site switch has been attempted, $U$ is the energy of the system, $\Gamma$ is the deviation from the target pattern (defined below), $T$ is the temperature of the system, and $T_{\Gamma}$ is an effective temperature that is used to weight the importance of target function fitting versus energy minimization. The energy $U$ is calculated by summing up all of the nonbonded segment-segment and segment-surface interactions. The deviation from the target pattern $\Gamma$ is calculated according to

$$
\Gamma_{i}=\frac{\sum_{(x, y)} \tau(x, y)}{L_{x} L_{y}},
$$

where $\tau(x, y)=1$ if the type of segment $(A$ or $B)$ present at $(x, y)$ during the simulation does not match the type of segment at $(x, y)$ in the target pattern, and 0 otherwise, and $L_{x} L_{y}$ is the area of the plane. The value of $\tau(x, y)$ is calculated only for the layer $z^{\prime}$ where the target pattern is applied. The summation on $\tau(x, y)$ is over all the $(x, y)$ coordinates in layer $z^{\prime}$ and is a measure of how well layer $z^{\prime}$ resembles the target pattern. We divide the summation by the area of the plane, $L_{x} L_{y}$, to scale the deviation between 0 (no deviation) and 1 (maximum deviation). Thus the term $\left(\Gamma_{\text {new }}-\Gamma_{\text {old }}\right)$ in the acceptance criterion (1) has the effect of minimizing the deviation from the target pattern. In our simulations, we choose $T_{\Gamma} / T=0.001$ in order to weigh the energy minimization term ten times more than the target function fitting term, as the energy minimization term has a magnitude of the order of $10^{\circ}$ and the target fitting term has a value of the order of magnitude $10^{-4}$. When no target pattern is applied, the term denoting the deviation from target pattern $\Gamma$ is always zero. In that case, the acceptance criterion depends only on energy minimization and is given by

$$
P_{\text {acc }}=\min \left[1, \exp \left\{-\frac{1}{k T}\left(U_{\text {new }}-U_{\text {old }}\right)\right\}\right] \text {. }
$$

The simulation proceeds in the following manner. For convenience, the initial configuration of the surface is an alternating sequence of 1 and 2 sites. The initial configuration of the chains is obtained by first placing "head" segments for all chains on random locations on the lattice. The second segments are placed on sites adjacent to the head segment on each of the chains, one by one. The third segments are placed on sites next to the second segment, one at a time, and this is repeated until the chains are all grown to the desired length. During this process, if there is no vacant site for adding a segment onto one end of the chain, the segment is added to the other end of the chain. If adding the segment to either end fails (because there is no vacant site), then the chains are moved in the box (using reptation, kink jump, and crankshaft moves) until a vacancy next to the chain in question is created.

For simulations, in which the target pattern is applied, there are three stages: initialization, equilibration, and production. In the initialization stage the system runs through 100000 MC steps moving the chains in the box with the segment-segment and segment-surface interactions switched off; the target pattern is not applied and the site switches are not attempted. This stage helps us avoid any bias that might arise due to the nature of the initial configuration of the chains. In the equilibration stage, the segment-segment and 
(a)

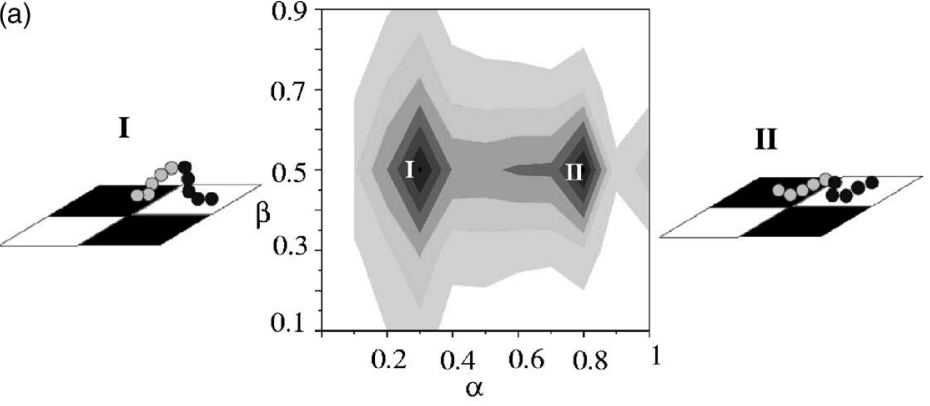

(c)

(b)

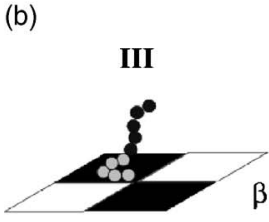

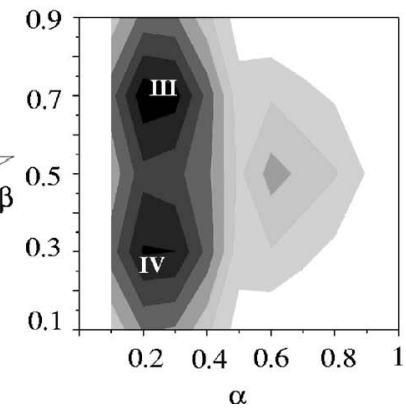

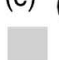

0.01

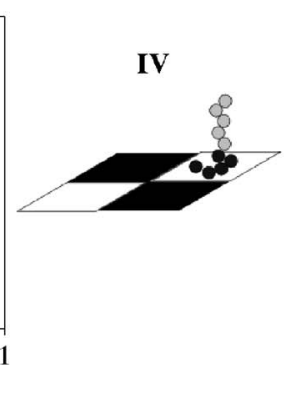

FIG. 1. Contour plots for loop conformation (I), flat conformation (II), and brush conformations (III and IV). $\alpha$ denotes the fraction of chain length adsorbed. $\beta$ denotes the ratio of the number of adsorbed segments on type 1 sites over the total number of adsorbed segments. The contour plots show the fraction of chains in the simulation box with the characteristics $\alpha$ and $\beta$. The values of the fractions are indicated by the colors shown in the key. segment-surface interactions are switched on, the target pattern is applied at the specified distance from the surface, and the chain segments are subjected to the usual assortment of moves with the site switches still turned off. During this stage a fraction of the polymers adsorb on the initial surface. Once the fraction of adsorbed segments reaches a certain value ( $2 \%$ of the segments in the system are adsorbed), the "production" stage or "design" stage starts. In the production stage the system goes through $8 \times 10^{6}$ MC steps, during which the standard chain moves are made, the target pattern is still applied at the specified distance from the surface, and the sites 1 and 2 are subjected to identity switches. We obtain data every $1000 \mathrm{MC}$ steps and calculate the averages of the properties described in Sec. II B. We take averages obtained from ten trials and compute the standard deviations. At the end of the simulation we analyze the pattern of sites 1 and 2 on the designed surface to determine the extent to which the pattern has been transferred from the adsorbed layers to the surface.

For the simulation involving no target pattern there are two stages: initialization and equilibration. The initialization stage is the same as mentioned above. In the equilibration stage, the segment-segment and segment-surface interactions are switched on, the chain segments are subjected to the MC moves, and the site switches are turned on; the target pattern is not applied. At the end of the simulation the copolymers in the adsorbed layer attain their energetically favorable conformations.

\section{B. Adsorption features}

To characterize the adsorption process, we monitor various properties of the system as a function of the number of MC steps. These include the extent of adsorption, as measured by the number of adsorbed segments of type $A$ and $B$, and the conformation of the chains near the surface during the adsorption process. The conformations of the adsorbed chains can be inferred from a contour plot depicting $\alpha$, the fraction of chain length adsorbed, and $\beta$, the ratio of the number of adsorbed segments on top of type 1 sites to the total number of adsorbed segments. An example of such a contour plot is given in Fig. 1. The plot in Fig. 1(a) represents a system where most of the chains are adsorbed either in a flat conformation or in a loop conformation. The plot in Fig. 1(b) depicts a situation where most of the chains are adsorbed in brush conformations. The rationale for this identification is the following. If a large fraction of chains are adsorbed in loop conformations, most of the segments will be extended from the surface with only a few segments lying in the $z=1$ layer, implying that $\alpha \leqslant 0.5$; due to symmetry of the copolymers, equal numbers of adsorbed segments will lie on top of type 1 and type 2 sites, implying that $\beta=0.5$ [region I in Fig. 1(a)]. If a large fraction of chains are adsorbed in flat conformations, most of the segments will lie in the $z=1$ layer, implying that $\alpha>0.5$; due to the symmetry of the diblock copolymers, equal numbers of adsorbed segments will lie on top of type 1 and type 2 sites, implying that $\beta=0.5$ [region II in Fig. 1(a)]. If a large fraction of chains are adsorbed in brush conformations, a small section of each chain (all of the same segment type) will be adsorbed on the surface $(\alpha<0.5)$ and the adsorbing segments of types $A$ and $B$ will likely adsorb on their preferred sites (since the segment-surface interactions $\epsilon_{A 1}$ and $\epsilon_{B 2}$ are attractive) making $\beta \gg 0.5$ [region III in Fig. 1(b)] or $\beta \ll 0.5$ [region IV in Fig. 1(b)].

\section{Calculation of the extent of pattern transfer}

In order to quantify the extent of pattern transfer we introduce a pattern transfer parameter PTP. ${ }^{15-17}$ The PTP of the copolymer at height $z$ from the surface is defined as

$$
\operatorname{PTP}(z)=\operatorname{PTP}_{A}(z)+\operatorname{PTP}_{B}(z)
$$




$$
\begin{aligned}
& \operatorname{PTP}_{A}(z)= \frac{\sum_{\left(x_{A}, y_{A}\right)} \phi_{A}(x, y, z)-\sum_{\left(x_{B}, y_{B}\right)} \phi_{A}(x, y, z)}{\sum_{(x, y)} \phi_{A}(x, y, z)+\sum_{(x, y)} \phi_{B}(x, y, z)}, \\
& \operatorname{PTP}_{B}(z)=\frac{\sum_{\left(x_{B}, y_{B}\right)} \phi_{B}(x, y, z)-\sum_{\left(x_{A}, y_{A}\right)} \phi_{B}(x, y, z)}{\sum_{(x, y)} \phi_{A}(x, y, z)+\sum_{(x, y)} \phi_{B}(x, y, z)},
\end{aligned}
$$

where $\left(x_{A}, y_{A}\right)$ and $\left(x_{B}, y_{B}\right)$ are the $x, y$ coordinates of the $A$ and $B$ segments, respectively, in the layer at which the target pattern is applied, and $\phi_{A}(x, y, z)$ and $\phi_{B}(x, y, z)$ are the average volume fraction of segments $A$ and $B$, respectively, at position $(x, y, z)$. In Eq. (5) the first term in the numerator is the sum of the volume fractions of $A$ segments in the $z$ plane, whose $(x, y)$ positions are the same as the positions of the $A$ segments in the layer $z^{\prime}$ where the target pattern is applied. The second term in the numerator represents the sum of the volume fractions of $A$ segments, whose positions are different from the positions of the $A$ segments in the layer $z^{\prime}$. In Eq. (6) the first term in the numerator is the sum of the volume fractions of $B$ segments in the $z$ plane, whose positions are the same as the positions of the $B$ segments in the layer $z^{\prime}$. The second term in the numerator stands for the sum of the volume fractions of $B$ segments in the $z$ plane whose positions are different from the positions of the $B$ segments in the layer $z^{\prime}$. The larger the value of the first terms in $\operatorname{PTP}_{A}(z)$ and $\operatorname{PTP}_{B}(z)$, the better the match between the pattern on layer $z$ and layer $z^{\prime}$. The larger the value of the second terms in $\operatorname{PTP}_{A}(z)$ and $\operatorname{PTP}_{B}(z)$, the greater the mismatch between the pattern on layer $z$ and layer $z^{\prime}$. Thus the numerators of $\operatorname{PTP}_{A}(z)$ and $\operatorname{PTP}_{B}(z)$ express how well the pattern formed by $A$ and $B$ segments, respectively, at a certain $z$ layer resembles the pattern formed in the layer $z^{\prime}$. The denominator is the sum of the volume fractions of all the $A$ and $B$ segments in layer $z$. The value of PTP ranges between -1 (complete negative pattern transfer) and 1 (complete positive pattern transfer).

When we apply the pattern at $z^{\prime}$, we calculate the PTP at all layers below $z^{\prime}$ to the surface. For example, when the pattern is applied at $z^{\prime}=3$ we calculate $\operatorname{PTP}(z=0)$, $\operatorname{PTP}(z=1)$, and $\operatorname{PTP}(z=2)$ and, by doing so, we can observe the pattern transfer through the adsorbed layers.

\section{RESULTS AND DISCUSSION}

When a target pattern is applied, the extent of pattern transfer is expected to be affected by the length of the copolymers, the distance from the surface where the target pattern is applied, and the size of the target pattern. In this section, we present and discuss the results from our simulations, showing the effect of variation in each of these parameters on the extent of pattern transfer. We consider chains of length 12,18 , and 24; checkerboard patterns with sizes $6 \times 6,12 \times 12$, and $24 \times 24$; and target patterns at distances from the surface of $z^{\prime}=1, z^{\prime}=2$, and $z^{\prime}=3$. For each of the chain lengths considered we compare the cases when a target pattern is applied to the case when no pattern is applied and the copolymers are free to attain their natural conformations. This helps us to (1) understand why a certain target pattern is

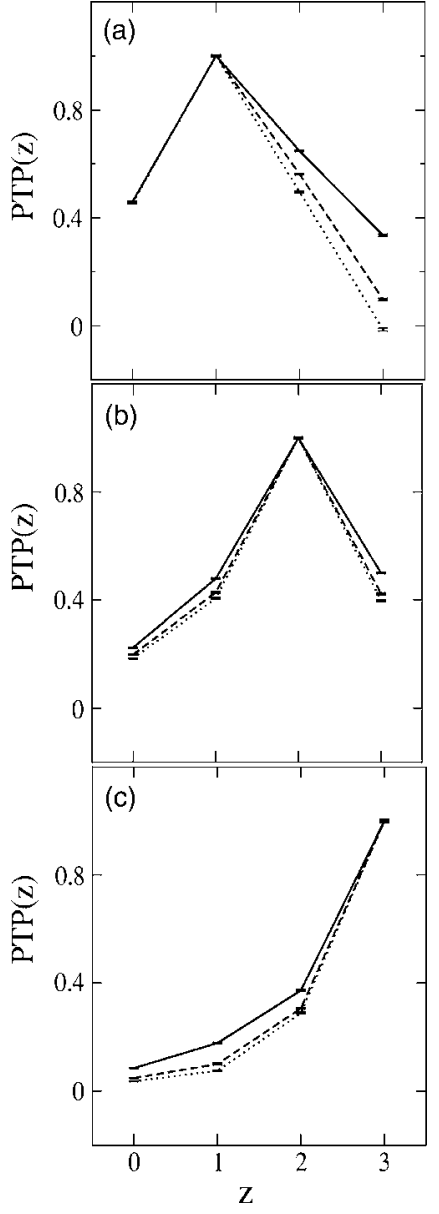

FIG. 2. Pattern transfer parameter $\operatorname{PTP}(z)$, for chain length $=12$, as a function of the distance from the surface $z$ for different target patterns: $6 \times 6$ (solid line), $12 \times 12$ (dashed line), and $24 \times 24$ (dotted line) applied at (a) $z^{\prime}=1$, (b) $z^{\prime}=2$, and (c) $z^{\prime}=3$.

transferred to the surface better than other patterns and (2) determine how the energy of the system, when the target pattern is applied, compares with the energy of the system when no pattern is applied. After the target pattern is transferred to the surface and the surface pattern is designed, we adsorb a new copolymer film in a random configuration (with the same chain length and interaction potentials) onto the designed surface to test if the designed surface can transfer the pattern back through the adsorbed layers, inducing the target pattern at the desired distance from the surface.

\section{A. Pattern transfer for different chain lengths}

In Fig. 2 we plot the PTP as a function of distance $z$ from the surface for chain length 12 when the pattern is applied at (a) $z^{\prime}=1$, (b) $z^{\prime}=2$, and (c) $z^{\prime}=3$ for different pattern sizes $(6 \times 6,12 \times 12,24 \times 24)$. When the target pattern is applied at $z^{\prime}=1$, the layer adjacent to the surface [Fig. 2(a)], the $\operatorname{PTP}(z=0)$ is approximately 0.46 for all the pattern sizes indicating that the pattern sizes do not affect the extent of pattern transfer in this case. When the target pattern is applied at $z^{\prime}=2$ [Fig. 2(b)], the $\operatorname{PTP}(z=0)$ for the $6 \times 6$ pattern is 0.24 while the $\operatorname{PTPs}(z=0)$ for patterns $12 \times 12$ and $24 \times 24$ are 0.19 and 0.18 , respectively, revealing that the $6 \times 6$ pattern is transferred to the surface with higher fidelity 
TABLE I. Pattern transfer parameter PTP $(z=0)$ for different chain lengths and pattern sizes.

\begin{tabular}{ccccc}
\hline \hline$z^{\prime}$ & Pattern & $N=12$ & $N=18$ & $N=24$ \\
\hline$z^{\prime}=1$ & $6 \times 6$ & 0.46 & 0.47 & 0.46 \\
& $12 \times 12$ & 0.46 & 0.49 & 0.50 \\
& $24 \times 24$ & 0.46 & 0.49 & 0.50 \\
$z^{\prime}=2$ & $6 \times 6$ & 0.24 & 0.29 & 0.32 \\
& $12 \times 12$ & 0.19 & 0.32 & 0.37 \\
& $24 \times 24$ & 0.18 & 0.32 & 0.39 \\
$z^{\prime}=3$ & $6 \times 6$ & 0.10 & 0.17 & 0.20 \\
& $12 \times 12$ & 0.04 & 0.17 & 0.23 \\
& $24 \times 24$ & 0.03 & 0.14 & 0.24 \\
\hline \hline
\end{tabular}

than the $12 \times 12$ and $24 \times 24$ patterns. When the target pattern is applied at $z^{\prime}=3$ [Fig. 2(c)] the $\operatorname{PTPs}(z=0)$ indicate that the $6 \times 6$ pattern $(0.10)$ is transferred to the surface better than the $12 \times 12(0.04)$ and $24 \times 24(0.03)$ patterns. The differences in the PTP values for the various patterns are significant since the error bars for all the PTP values discussed in this paper were of the order of 0.001. The $\operatorname{PTPs}(z=0)$ for all chain lengths and pattern sizes are also tabulated in Table I.

For chain length 12, when the pattern is applied at $z^{\prime}=1$, the reason that all the patterns are transferred equally well to the surface has to do with the energy. Figure 3 shows the average difference $\Delta U^{\prime}$ between the equilibrium energy per segment, when a target pattern is applied and that when no target pattern is applied, keeping all of the parameters the same. The filled black circles in Fig. 3(a) show that at $z^{\prime}=1$ the $\Delta U^{\prime}$ for the $6 \times 6$ pattern $(0.00396 k T)$, the 12 $\times 12$ pattern $(0.0072 k T)$, and the $24 \times 24$ pattern $(0.0094 k T)$ are all less than $0.01 k T$. This means that when the target pattern is applied at $z^{\prime}=1$ regardless of the pattern size, the energy of the system is close to the case when no pattern is applied. In other words, we do not have to force the chains into unfavorable conformations when we apply a target pattern at $z^{\prime}=1$ for this chain length. When the pattern is applied at $z^{\prime}=2$ or $z^{\prime}=3$, the $6 \times 6$ pattern is transferred to the surface better than the $12 \times 12$ and $24 \times 24$ patterns. This is because the $\Delta U^{\prime}$ for the $6 \times 6$ pattern is lower than the $\Delta U^{\prime}$ for the $12 \times 12$ and $24 \times 24$ patterns [Fig. 3(a)]. This means that when the $12 \times 12$ and $24 \times 24$ patterns are applied at $z^{\prime}=2$ or $z^{\prime}=3$, the chains are forced to be in unfavorable conformations and the energy is high relative to the case when no target pattern is applied. The chains try to resist these unfavorable conformations and the pattern transfer is reduced.

Thus in the case of chain length 12 , for all $z^{\prime}$ considered, the $6 \times 6$ pattern helps the chains of length 12 adsorb in favorable conformations and maintain the energy of the system close to the energy of the system when no pattern is applied. When the $12 \times 12$ and $24 \times 24$ patterns are applied we force the copolymer chains to adopt conformations that increase the energy; the chains resist those conformations and in turn reduce the pattern transfer.

For chain length 18 , when the pattern is applied at $z^{\prime}=1$ or $z^{\prime}=2$, the $12 \times 12$ and $24 \times 24$ patterns are trans-
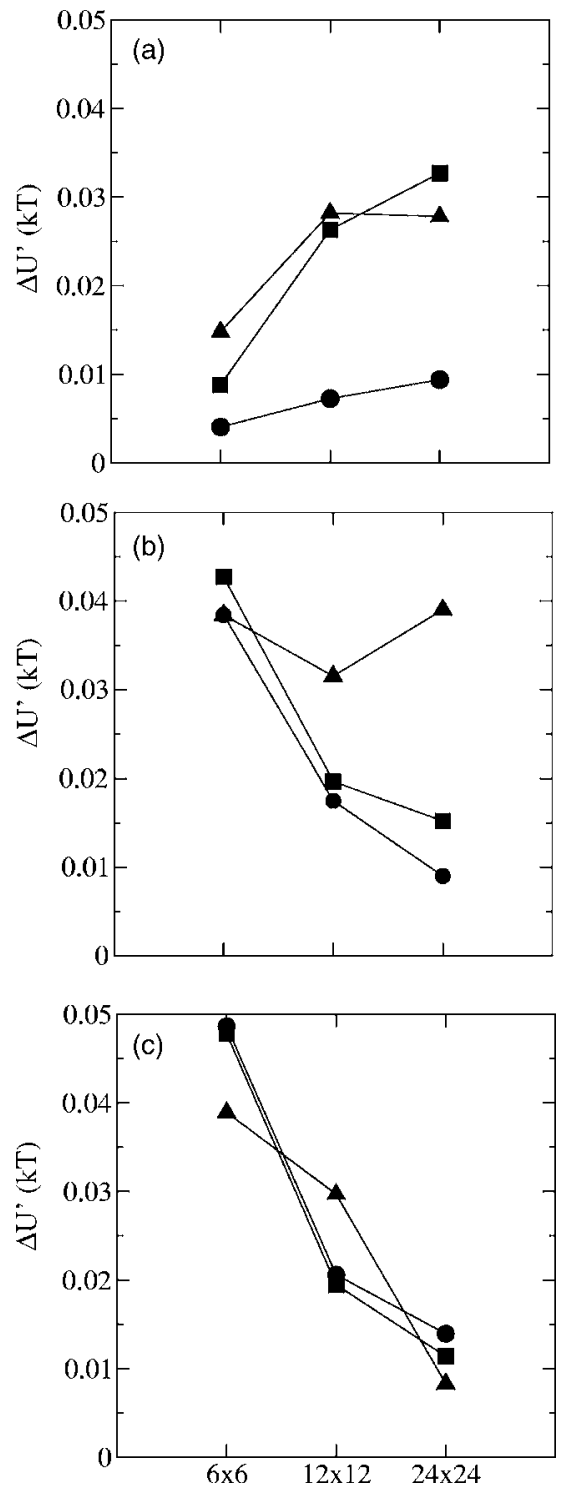

FIG. 3. The difference $\Delta U^{\prime}$ between the system internal energy when the target pattern is applied and when no target pattern is applied for different target pattern sizes at chain lengths (a) 12, (b) 18, and (c) 24. The circles represent $z^{\prime}=1$, squares represent $z^{\prime}=2$, and triangles represent $z^{\prime}=3$. The lines are drawn as a guide to the eye.

ferred to the surface slightly better than the $6 \times 6$ pattern (Table I). When the target pattern is applied at $z^{\prime}=3$, the $6 \times 6$ and $12 \times 12$ patterns are transferred to the surface better than the $24 \times 24$ pattern (Table I). For $z^{\prime}=1$, the $12 \times 12$ and $24 \times 24$ patterns are transferred to the surface better than the $6 \times 6$ pattern because the $\Delta U^{\prime}$ for the $12 \times 12$ pattern $(0.018 \mathrm{kT})$ and the $24 \times 24$ pattern $(0.009 \mathrm{kT})$ are lower than the $\Delta U^{\prime}$ for the $6 \times 6(0.038 \mathrm{kT})$ pattern [circles in Fig. 3(b)]. This means that the $12 \times 12$ and $24 \times 24$ patterns help the chains form conformations that bring the energy of the system close to the energy when no pattern is applied. Contour plots, such as the ones in Fig. 4, help us to understand the conformations that the adsorbed chains adopt. As mentioned previously in Sec. II B, the conformations of the adsorbed chains are deduced from $\alpha$, the fraction of chain length adsorbed, and $\beta$, the ratio of the number of adsorbed segments on top of type 1 sites to the total number of adsorbed seg- 
$6 \times 6$
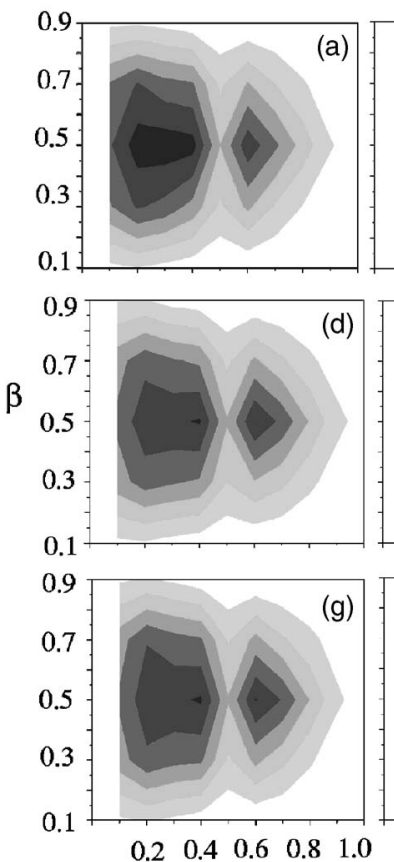

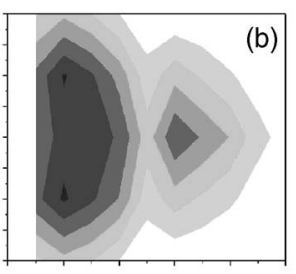

d)
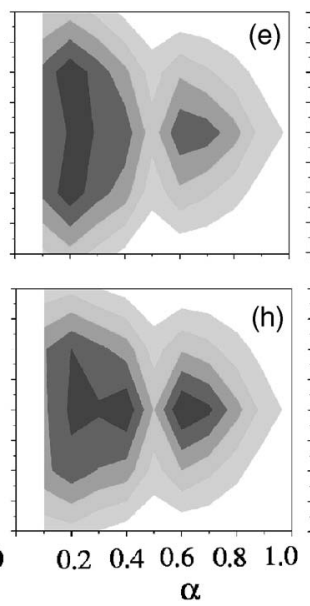

(h)
$24 \times 24$
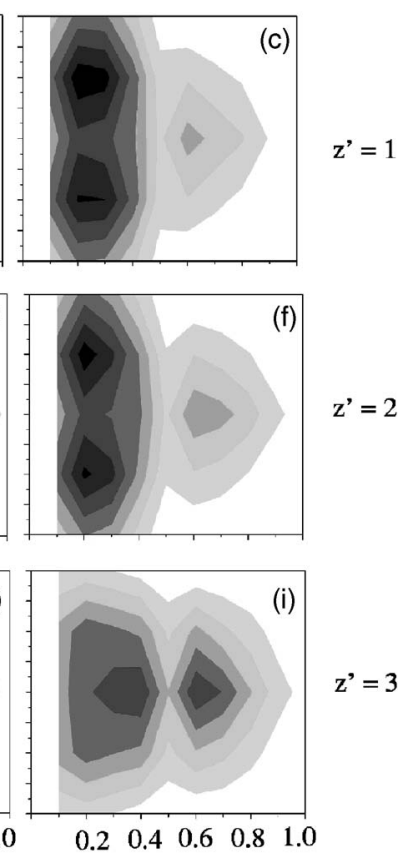

FIG. 4. Contour plot for chain length 18, showing $\alpha$, the fraction of chain length adsorbed, and $\beta$, the ratio of the number of adsorbed segments on top of type 1 sites to the total number of adsorbed segments. The values of the fractions are indicated by the colors in the key on Fig. 1(c). ments. When a large fraction of chains adsorb in loop conformations, $\alpha \leqslant 0.5$ and $\beta=0.5$. When a large fraction of chains adsorb in flat conformations, $\alpha>0.5$ and $\beta=0.5$. When a large fraction of chains adsorb in brush conformations, $\alpha<0.5$ and $\beta \gg 0.5$ or $\beta \ll 0.5$. For clarity, data from such contour plots are tabulated in Table II which shows the conformations of the adsorbed chains for all the chain lengths and all pattern sizes applied at $z^{\prime}=1, z^{\prime}=2$, and $z^{\prime}=3$, and when no pattern is applied.

For chain length 18 and $z^{\prime}=1$, the comparison of the adsorbed chains conformations with those when no pattern is applied can tell us why the $12 \times 12$ and $24 \times 24$ patterns get transferred to the surface better than the $6 \times 6$ pattern. When no pattern is applied for chain length 18 the chains adsorb in conformations similar to the case when the $6 \times 6$ and $12 \times 12$ patterns are applied at $z^{\prime}=1$ (Table II). However, for the $6 \times 6$ pattern and $z^{\prime}=1, \Delta U^{\prime}$ is not low even though the conformations are similar to those when no pattern is applied [Fig. 3(b)]. On the other hand for the $24 \times 24$ pattern and $z^{\prime}=1, \Delta U^{\prime}$ is very low, although the conformations are different from those when no pattern is applied. Thus it is clear that there can be many conformations of the adsorbed chains leading to a $U^{\prime}$ close to the $U^{\prime}$ when no pattern is applied. For $z^{\prime}=2$, the $12 \times 12$ and $24 \times 24$ patterns are transferred to the surface better than the $6 \times 6$ pattern because the $\Delta U^{\prime}$ for the $12 \times 12(0.020 k T)$ and $24 \times 24(0.015 k T)$ patterns are lower than the $\Delta U^{\prime}$ for the $6 \times 6(0.043 k T)$ pattern [squares in Fig. 3(b)]. The conformations of the adsorbed chains when no pattern is applied for chain length 18 is similar to the case when the $6 \times 6$ pattern is applied at $z^{\prime}=2$ (Table II), but the $\Delta U^{\prime}$ is not low for the $6 \times 6$ pattern. As was the case for $z^{\prime}=1$, the conformations of the adsorbed chains for the pattern that has the lowest value of $\Delta U^{\prime}$ are not similar to the conformations of the adsorbed chains when no pattern is

TABLE II. Fraction of chains in the system adsorbed in different conformations.

\begin{tabular}{|c|c|c|c|c|c|c|c|c|c|c|}
\hline \multirow[b]{2}{*}{$z^{\prime}$} & \multirow[b]{2}{*}{ Pattern } & \multicolumn{3}{|c|}{$N=12$} & \multicolumn{3}{|c|}{$N=18$} & \multicolumn{3}{|c|}{$N=24$} \\
\hline & & Flat & Loop & Brush & Flat & Loop & Brush & Flat & Loop & Brush \\
\hline \multirow[t]{3}{*}{$z^{\prime}=1$} & $6 \times 6$ & 0.09 & 0.09 & 0.04 & 0.05 & 0.14 & 0.20 & 0.06 & 0.25 & 0.19 \\
\hline & $12 \times 12$ & 0.08 & 0.09 & 0.09 & 0.06 & 0.11 & 0.23 & 0.06 & 0.22 & 0.19 \\
\hline & $24 \times 24$ & 0.06 & 0.09 & 0.13 & 0.04 & 0.09 & 0.36 & 0.04 & 0.15 & 0.38 \\
\hline \multirow[t]{3}{*}{$z^{\prime}=2$} & $6 \times 6$ & 0.10 & 0.09 & 0.03 & 0.07 & 0.12 & 0.22 & 0.07 & 0.22 & 0.18 \\
\hline & $12 \times 12$ & 0.10 & 0.08 & 0.03 & 0.06 & 0.11 & 0.25 & 0.07 & 0.21 & 0.29 \\
\hline & $24 \times 24$ & 0.09 & 0.07 & 0.05 & 0.05 & 0.09 & 0.30 & 0.05 & 0.16 & 0.41 \\
\hline \multirow[t]{4}{*}{$z^{\prime}=3$} & $6 \times 6$ & 0.10 & 0.08 & 0.04 & 0.07 & 0.12 & 0.22 & 0.07 & 0.22 & 0.17 \\
\hline & $12 \times 12$ & 0.10 & 0.08 & 0.05 & 0.07 & 0.11 & 0.21 & 0.07 & 0.21 & 0.18 \\
\hline & $24 \times 24$ & 0.10 & 0.09 & 0.03 & 0.07 & 0.11 & 0.19 & 0.07 & 0.19 & 0.31 \\
\hline & No pattern & 0.09 & 0.07 & 0.04 & 0.07 & 0.14 & 0.23 & 0.07 & 0.21 & 0.19 \\
\hline
\end{tabular}


applied. For $z^{\prime}=3$ the $\Delta U^{\prime}$ for the $6 \times 6(0.038 k T)$ and the $12 \times 12(0.032 \mathrm{kT})$ patterns are lower than the $\Delta U^{\prime}$ for the $24 \times 24(0.039 k T)$ pattern [triangles in Fig. 3(b)]. The conformations of the adsorbed chains of chain length 18 when no pattern is applied (Table II) are similar to the cases when the $6 \times 6$ and $12 \times 12$ patterns are applied at $z^{\prime}=3$ (Table II), and the $\Delta U^{\prime}$ for the $6 \times 6$ and $12 \times 12$ patterns are the lowest. Thus the conformations of the adsorbed chains for the pattern that has the lowest $\Delta U^{\prime}$ are similar to the conformations of the adsorbed chains when no pattern is applied. However, since the same trend was not observed for $z^{\prime}=1$ and $z^{\prime}=2$ cases we cannot conclusively relate the $\Delta U^{\prime}$ and the conformations.

Thus in the case of chain length 18, the patterns that have low $\Delta U^{\prime}$ get transferred to the surface better. In order to achieve a low $\Delta U^{\prime}$ the conformations of the adsorbed chains do not necessarily have to match the case when no pattern is applied.

For the longest chains (chain length 24) when the target pattern is applied at $z^{\prime}=1$, the $12 \times 12$ and $24 \times 24$ patterns are transferred to the surface better than the $6 \times 6$ pattern (Table I). For $z^{\prime}=2$ or $z^{\prime}=3$, the $24 \times 24$ pattern is transferred to the surface better than the $6 \times 6$ and $12 \times 12$ patterns (Table I). Analysis of the data in Tables I and II and Fig. 3(c) similar to those described above for the chain lengths 12 and 18 cases reveals the following trends. For chain length 24, when the $12 \times 12$ pattern is applied at $z^{\prime}=2$ and $z^{\prime}=3$ and when the $6 \times 6$ pattern is applied at $z^{\prime}=1$ the chains adsorb in conformations similar to the no-pattern case. However, for all three cases, $z^{\prime}=1, z^{\prime}=2$, and $z^{\prime}=3$, the $24 \times 24$ pattern has the energy closest to the energy when no pattern is applied. Thus we can conclude that even if the conformations are not similar to the no-pattern case, the energy of the system can be maintained close to the energy when no pattern is imposed.

\section{B. Pattern transfer for different pattern sizes}

When the $6 \times 6$ pattern is applied at $z^{\prime}=1$, the $\operatorname{PTPs}(z$ $=0)$ are almost equal for all chain lengths (Table I), implying that the $6 \times 6$ pattern is transferred well, regardless of the chain length. When the $6 \times 6$ pattern is applied at $z^{\prime}=2$ and $z^{\prime}=3$, the pattern transfer to the surface improves as chain length increases. This is because longer chains have a higher fraction of adsorbing chains forming loop and brush conformations than the shorter chains (Table II). When the adsorbing chains form loops, a part of the chain extends to $z>1$ layers and thus bridges the pattern between the different layers. In order to transfer the pattern well between the layers we need to maintain a connectivity between the different layers. Therefore the systems with chains of length 24 , which have a higher fraction of chains adsorbing in loop and brush conformations, transfer the patterns to the surface better than the systems with chain lengths 12 and 18 .

When the $12 \times 12$ pattern is applied at $z^{\prime}=1, z^{\prime}=2$, or $z^{\prime}=3$, the values of the the $\operatorname{PTPs}(z=0)$ in Table I reveal that the $12 \times 12$ pattern gets transferred to the surface best for chain length 24 . When the $24 \times 24$ pattern is applied at $z^{\prime}=1$, the $\operatorname{PTP}(z=0)$ is higher for chain lengths 24 and 18
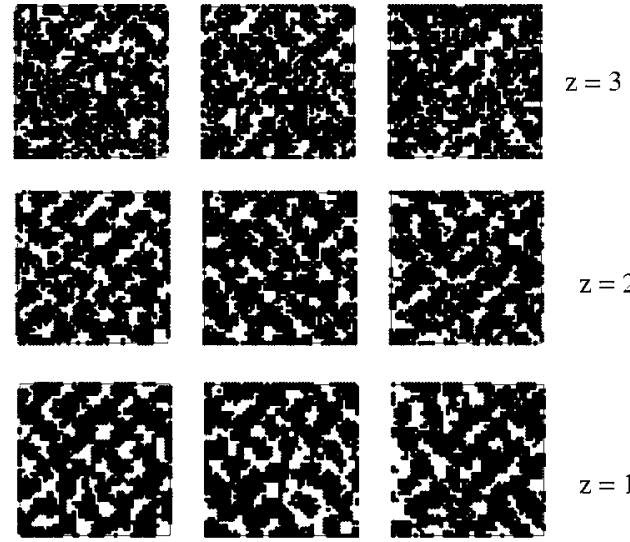

$z=2$
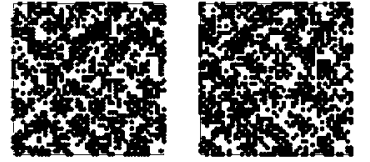

(a) $z^{\prime}=1$

(b) $z^{\prime}=2$

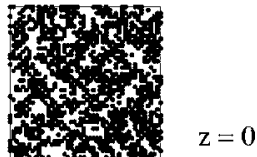

(c) $z^{\prime}=3$

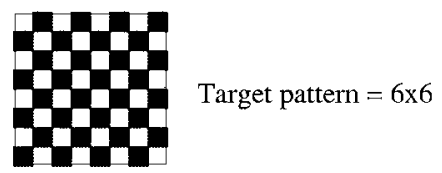

FIG. 5. The designed surfaces $(z=0)$ when the $6 \times 6$ pattern is applied at (a) $z^{\prime}=1$, (b) $z^{\prime}=2$, and (c) $z^{\prime}=3$ with $T_{\Gamma} / T=0.001$. The $6 \times 6$ target pattern is shown for comparison. Also shown are the patterns observed in the bulk at $z=1,2$, and 3 during the test stage when diblock copolymers of chain length 12 are adsorbed on the designed surfaces.

than for chain length 12 (Table I). For $z^{\prime}=2$ and $z^{\prime}=3$, the $\operatorname{PTP}(z=0)$ is again highest for chain length 24 (Table I), implying that the pattern transfer to the surface is best for longer chain lengths. The reason why chain length 24 has the best pattern transfer is the same as discussed for the $6 \times 6$ pattern.

\section{Copolymer bulk adsorbed on the designed surfaces}

So far, we have picked a target pattern, biased the adsorbed copolymer film to adopt that target pattern at a desired distance from the surface, let the pattern be transferred through the adsorbed layers to the surface, and designed the surface in response to the target pattern. The next question to be asked is the following: If a bulk of copolymers in a random configuration is placed on the designed surface, can the designed surface induce the target pattern in the adsorbing layers? Answering this question will also reveal if we can determine beforehand what pattern must be present on the surface to induce adsorbing copolymer layers to attain a target pattern at a certain distance from the surface.

In order to answer the question posed above, we allow the designed surfaces obtained in each of the cases discussed in Sec. III B to adsorb $A B$ diblock copolymer bulk initially in random configurations. We term this "the test stage." Our purpose here is to test if the designed surfaces can induce the target pattern that was used originally to form the surface pattern. Figure 5 shows how the $A B$ copolymer chains of length 12 respond to the surfaces designed for an $A B$ copoly- 

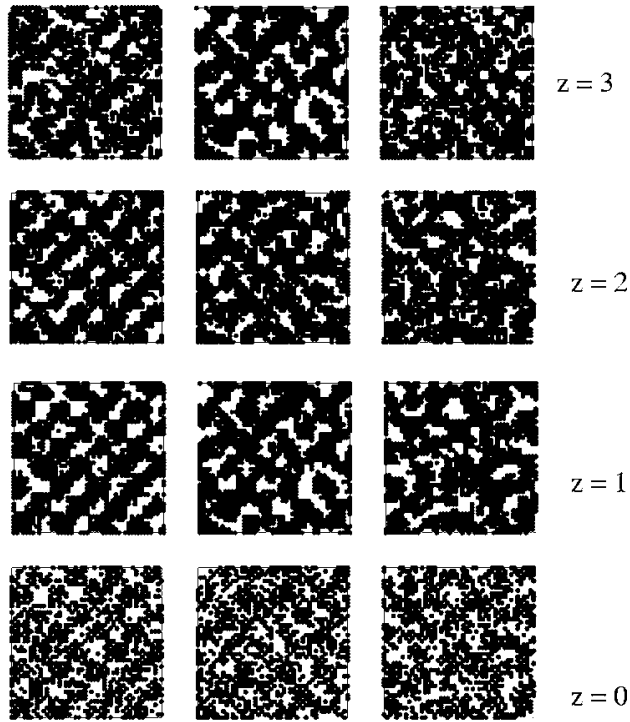

(a) $z^{\prime}=1$
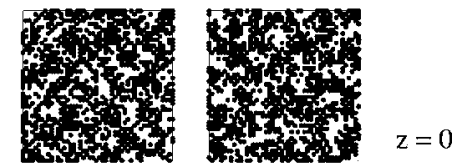

(b) $z^{\prime}=2$

(c) $z^{\prime}=3$
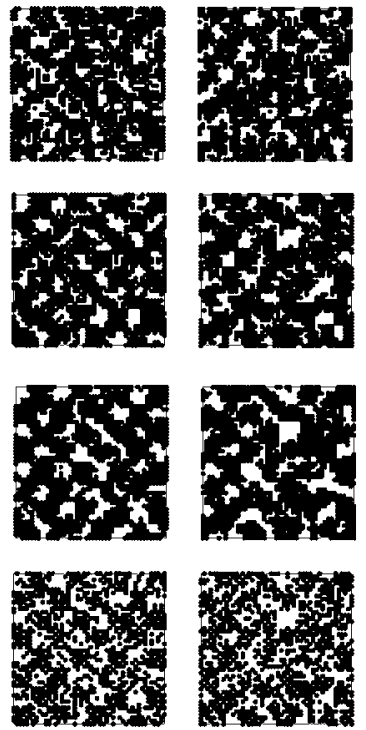

(a) $z^{\prime}=1$

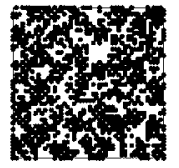

(b) $z^{\prime}=2$
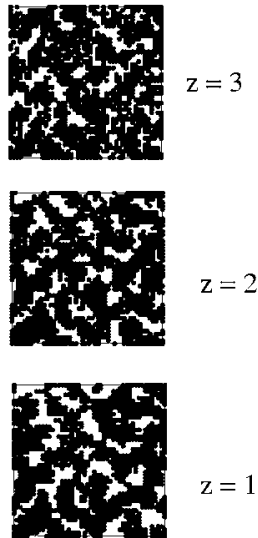

(c) $z^{\prime}=$

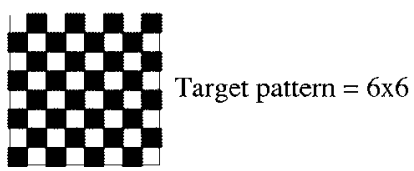

FIG. 6. The designed surfaces $(z=0)$ when the $6 \times 6$ pattern is applied at (a) $z^{\prime}=1$, (b) $z^{\prime}=2$, and (c) $z^{\prime}=3$ with $T_{\Gamma} / T=0.0001$. The $6 \times 6$ target pattern is shown for comparison. Also shown are the patterns observed in the bulk at $z=1,2$, and 3 during the test stage when diblock copolymers of chain length 12 are adsorbed on the designed surfaces.

mer film of chain length 12 when the $6 \times 6$ target pattern has been applied at (a) $z^{\prime}=1$, (b) $z^{\prime}=2$, and (c) $z^{\prime}=3$. The designed surface patterns at $z=0$ are also shown. The data in Fig. 5 indicate that the $6 \times 6$ target pattern was not induced at any distance from the surface. Similarly, for all chain lengths the designed surfaces for the other patterns, $12 \times 12$ and $24 \times 24$, did not induce any of the target patterns in the copolymer bulk (not shown here for brevity). This is because the patterns applied in the Secs. III A and III B allow the copolymer chains to have conformations that are not necessarily similar to the conformations when no pattern is applied. When we bias the copolymer bulk to attain a target pattern at a certain distance from the surface, we force the chains to adopt conformations that will help fit the target pattern, which does not correspond to their natural conformation. When there is no bias and the copolymer chains adsorb on the designed surface, they are free to have conformations close to their natural conformations and therefore do not form the target pattern we wanted them to attain.

To ensure that the results above were not an artifact resulting from the low value of weighting factor $T_{\Gamma} / T(=0.001)$ used in Eq. (2) to weight the energy minimization term versus the target function fitting term, we run all the above simulations for all chain lengths and all pattern sizes at two other values of the weighting factor: 0.0001 and 0.00001 . When $T_{\Gamma} / T=0.001$, the weighting factor used in the results shown so far, the target function fitting term is weighted $1 / 10$ the energy minimization term in the acceptance criterion. When $T_{\Gamma} / T=0.0001$ and $T_{\Gamma} / T=0.00001$ the target function fitting term is weighted equal to and ten times
FIG. 7. The designed surfaces $(z=0)$ when the $6 \times 6$ pattern is applied at (a) $z^{\prime}=1$, (b) $z^{\prime}=2$, and (c) $z^{\prime}=3$ with $T_{\Gamma} / T=0.00001$. The $6 \times 6$ target pattern is shown for comparison. Also shown are the patterns observed in the bulk at $z=1,2$, and 3 during the test stage when diblock copolymers of chain length 12 are adsorbed on the designed surfaces.

the energy minimization term, respectively, in the acceptance criterion. Figures 6 and 7 show how the $A B$ copolymer chains of length 12 respond to the surfaces designed for an $A B$ copolymer film of chain length 12 when the $6 \times 6$ target pattern has been applied at (a) $z^{\prime}=1$, (b) $z^{\prime}=2$, and (c) $z^{\prime}=3$ using values of weighting factors $T_{\Gamma} / T=0.0001$ and $T_{\Gamma} / T=0.00001$. In both cases we see that the target pattern is not induced in the copolymer bulk. This implies that irrespective of the weighting factor while designing the surface pattern, we do not induce the target pattern in the bulk.

In order to compare the system at the test stage to the system during the design, we show the designed surfaces $(z=0)$ and the patterns observed in the bulk at $z=1,2$, and 3 during the design stage when the $6 \times 6$ pattern is applied in a bulk of diblock copolymers of chain length 12 at (a) $z^{\prime}=1$, (b) $z^{\prime}=2$, and (c) $z^{\prime}=3$ with $T_{\Gamma} / T=0.00001$ in Fig. 8. The pattern obtained in the $z=1$ layer of the bulk during the surface design when a pattern is applied at $z^{\prime}=1$ (Fig. 8) is not the same as the pattern obtained in the $z=1$ layer of the bulk during the test stage (case $z^{\prime}=1$ in Fig. 7).

In Table III we tabulate the average equilibrium $\Delta U^{\prime}$ for the chains in the system: (1) during surface design, i.e., when the target pattern is applied at $z^{\prime}$ and the surface reorganizes itself in response to the target pattern, and (2) in the test stage, i.e., after the new copolymer bulk has been adsorbed and equilibrated on the designed surface. The data in part (1) of Table III are the same as the data plotted in Fig. 3, i.e., during surface design when the target pattern is applied at $z^{\prime}$ with $T_{\Gamma} / T=0.001$. In part (2) of Table III, the cases where the $\Delta U^{\prime}$ values are smaller than the corresponding $\Delta U^{\prime}$ val- 


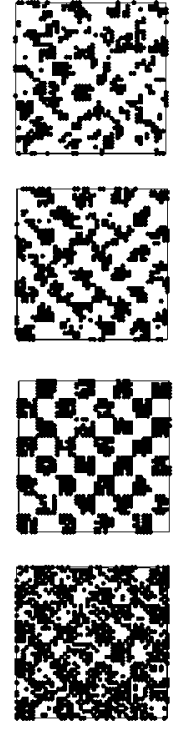

(a) $z^{\prime}=1$
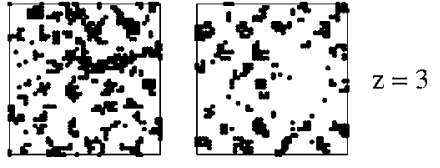

a
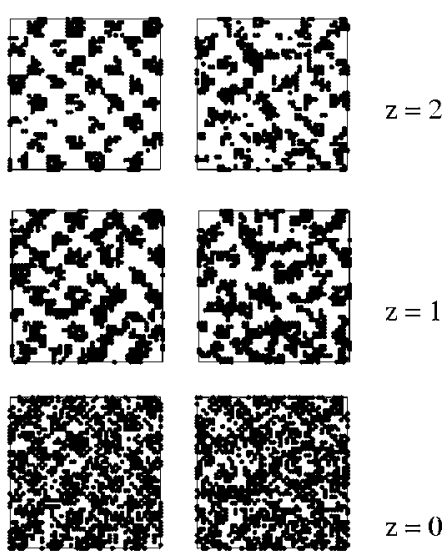

(b) $z^{\prime}=2$

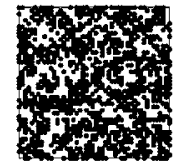

(c) $z^{\prime}=3$

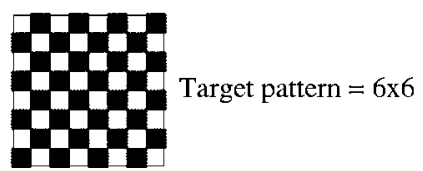

FIG. 8. The designed surfaces $(z=0)$ and the patterns observed in the bulk at $z=1,2$, and 3 during the design stage when the $6 \times 6$ pattern is applied in a bulk of diblock copolymers of chain length 12 at (a) $z^{\prime}=1$, (b) $z^{\prime}=2$, and (c) $z^{\prime}=3$ with $T_{\Gamma} / T=0.00001$.

ues in part (1) are shown in bold. These cases (shown in bold) during the test stage have energy closer to the energy of the natural conformations than the corresponding cases during the suface design. This reveals that for most cases when we do not force a target pattern on the adsorbed layers and adsorb the copolymer chains on the designed surface (test stage), the system prefers to attain an energy that is close to that of their natural conformations and therefore do not induce the target pattern in the adsorbed layers.

\section{SUMMARY}

We performed lattice Monte Carlo simulations to study how symmetric diblock copolymers transfer a pattern through the adsorbed layers to the surface. We applied a target pattern in the copolymer film at a certain height above the surface, performed chain moves to maintain the target pattern, and allowed the surface to rearrange itself in response to the target pattern. The attractive interactions between the copolymer segments and surface $\left(\epsilon_{A 1}\right.$ and $\left.\epsilon_{B 2}\right)$ and the attractive interactions between the like segments of the copolymer $\left(\epsilon_{A A}\right.$ and $\left.\epsilon_{B B}\right)$ enabled the surface to rearrange, forming a pattern similar to the target pattern. We varied the chain length, pattern size, and the height above the surface $\left(z^{\prime}\right)$ where the pattern was applied to study their effect on pattern transfer.

For constant chain length, the pattern that brings the energy of the system close to the energy when no pattern is applied is transferred to the surface the best. For the pattern that is transferred the best, the conformations of the adsorbed chains are not necessarily the same as when no pattern is applied. This shows that there can be many conformations that can bring the energy of the system close to that when no pattern is applied. For constant pattern size, increasing the chain length results in an increase in the extent of pattern transfer to the surface. This is because longer chains are able to adsorb in the form of brushes and loops that help transfer the pattern between adsorbed layers down to the surface.

After designing the surfaces, we tested if the surfaces could induce the target pattern back into the adsorbed layers of a new copolymer bulk in a random configuration. We found that for all the cases the surface pattern was not induced back into the adsorbed layers. This is because the target pattern we applied in the bulk when designing the surfaces did not let the chains attain energy close to that of their natural conformations.

\section{ACKNOWLEDGMENTS}

This work was supported by the Office of Energy Research, Basic Sciences, Chemical Science Division of the U.S. Department of Energy under Grant No. DE-FG0591ER14181.

TABLE III. Average equilibrium $\Delta U^{\prime}$ for different chain lengths and pattern sizes (1) during surface design and (2) in the test stage.

\begin{tabular}{|c|c|c|c|c|c|c|c|}
\hline \multirow[b]{2}{*}{$z^{\prime}$} & \multirow[b]{2}{*}{ Pattern } & \multicolumn{3}{|c|}{ (1) } & \multicolumn{3}{|c|}{ (2) } \\
\hline & & $N=12$ & $N=18$ & $N=24$ & $N=12$ & $N=18$ & $N=24$ \\
\hline \multirow[t]{3}{*}{$z^{\prime}=1$} & $6 \times 6$ & 0.0041 & 0.0384 & 0.0486 & 0.0061 & 0.0025 & 0.0028 \\
\hline & $12 \times 12$ & 0.0073 & 0.0175 & 0.0206 & 0.0013 & 0.0120 & 0.0150 \\
\hline & $24 \times 24$ & 0.0094 & 0.0089 & 0.0139 & 0.0044 & 0.0089 & 0.0014 \\
\hline \multirow[t]{3}{*}{$z^{\prime}=2$} & $6 \times 6$ & 0.0088 & 0.0427 & 0.0478 & 0.0016 & 0.0220 & 0.0190 \\
\hline & $12 \times 12$ & 0.0263 & 0.0196 & 0.0195 & 0.0077 & 0.0042 & 0.0190 \\
\hline & $24 \times 24$ & 0.0327 & 0.0152 & 0.0114 & 0.0015 & 0.0041 & 0.0150 \\
\hline \multirow[t]{3}{*}{$z^{\prime}=3$} & $6 \times 6$ & 0.0148 & 0.0384 & 0.0389 & 0.0014 & 0.0053 & 0.0089 \\
\hline & $12 \times 12$ & 0.0282 & 0.0315 & 0.0297 & 0.0016 & 0.0087 & 0.0097 \\
\hline & $24 \times 24$ & 0.0278 & 0.0390 & 0.0083 & 0.0012 & 0.0096 & 0.0082 \\
\hline
\end{tabular}


${ }^{1}$ S. Walheim, E. Schaffer, J. Mlynek, and U. Steiner, Science 283, 520 (1999).

${ }^{2}$ R. J. Todd, R. D. Johnson, and F. H. Arnold, J. Chromatogr., A 662, 13 (1994).

${ }^{3}$ T. L. Morkved, M. Lu, A. M. Urbas, E. E. Ehrichs, H. M. Jaeger, P. Mansky, and T. P. Russell, Science 273, 931 (1996).

${ }^{4}$ I. Norde, Surface and Interfacial Aspects of Biomedical Applications (Plenum, New York, 1995).

${ }^{5}$ R. F. Service, Science 270, 230 (1995).

${ }^{6}$ J. K. Cox, A. Eisenberg, and R. B. Lennox, Curr. Opin. Colloid Interface Sci. 4, 52 (1999)

${ }^{7}$ M. J. Fasolka and A. M. Mayes, Annu. Rev. Mater. Res. 31, 323 (2001).

${ }^{8}$ L. Rockford, Y. Liu, P. Mansky, T. P. Russell, M. Yoon, and S. G. J. Mochrie, Phys. Rev. Lett. 82, 2602 (1999).

${ }^{9}$ L. Rockford, S. G. J. Mochrie, and T. P. Russell, Macromolecules 34, 1487 (2001).

${ }^{10}$ S. O. Kim, H. H. Solak, M. P. Stoykovich, N. J. Ferrier, J. J. de Pablo, and P. F. Nealey, Nature (London) 424, 411 (2003).

${ }^{11}$ X. M. Yang, R. D. Peters, P. F. Nealey, H. H. Solak, and F. Cerrina, Macromolecules 33, 9575 (2000)
${ }^{12}$ J. Heier, J. Genzer, E. J. Kramer, F. S. Bates, S. Walheim, and G. Krausch, J. Chem. Phys. 111, 11101 (1999).

${ }^{13}$ D. Petera and M. Muthukumar, J. Chem. Phys. 107, 9640 (1997).

${ }^{14}$ D. Petera and M. Muthukumar, J. Chem. Phys. 109, 5101 (1998).

${ }^{15}$ J. Genzer, Macromol. Theory Simul. 11, 481 (2002).

${ }^{16}$ J. Genzer, J. Chem. Phys. 115, 4873 (2001)

${ }^{17}$ J. Genzer, Phys. Rev. E 63, 022601 (2001).

${ }^{18}$ J. J. Semler and J. Genzer, J. Chem. Phys. 119, 5275 (2003).

${ }^{19}$ J. J. Semler and J. Genzer, Macromol. Theory Simul. 13, 219 (2004).

${ }^{20}$ A. Jayaraman, C. K. Hall, and J. Genzer, Phys. Rev. Lett. 94, 078103 (2005).

${ }^{21}$ Y. A. Kriksin, P. G. Khalatur, and A. R. Khokhlov, J. Chem. Phys. 122, 114703 (2005).

${ }^{22}$ H. Chen and A. Chakrabarti, J. Chem. Phys. 108, 6897 (1998).

${ }^{23}$ C. Seok, K. F. Freed, and I. Szleifer, J. Chem. Phys. 112, 6452 (2000).

${ }^{24}$ C. Seok, K. F. Freed, and I. Szleifer, J. Chem. Phys. 112, 6443 (2000).

${ }^{25}$ K. T. Thomson and K. E. Gubbins, Langmuir 16, 5761 (2000).

${ }^{26}$ R. L. McGreevy, J. Phys.: Condens. Matter 13, 877 (2001).

${ }^{27}$ R. L. McGreevy and L. Pusztai, Electrochim. Acta 43, 1349 (1998). 